\title{
Fearful faces have a sensory advantage in the competition for awareness
}

Article

Accepted Version

Hedger, N., Adams, W. J. and Garner, M. (2015) Fearful faces have a sensory advantage in the competition for awareness. Journal of Experimental Psychology-Human Perception and Performance, 41 (6). pp. 1748-1757. ISSN 0096-1523 doi: https://doi.org/10.1037/xhp0000127 Available at https://centaur.reading.ac.uk/88355/

It is advisable to refer to the publisher's version if you intend to cite from the work. See Guidance on citing.

To link to this article DOI: http://dx.doi.org/10.1037/xhp0000127

Publisher: APA PsycNET

All outputs in CentAUR are protected by Intellectual Property Rights law, including copyright law. Copyright and IPR is retained by the creators or other copyright holders. Terms and conditions for use of this material are defined in the End User Agreement.

\section{www.reading.ac.uk/centaur}

\section{CentAUR}

Central Archive at the University of Reading 
Reading's research outputs online 
${ }^{a}$ Psychology, University of Southampton, Southampton, SO17 1BJ, UK

${ }^{\mathrm{b}}$ Clinical and Experimental Sciences, Faculty of Medicine, University of Southampton, Southampton, SO17 1BJ, UK

6

7

10

1

2

3

4 Corresponding author: Nicholas Hedger.

5 Email: naah1g08@soton.ac.uk

6 Phone: +447742142858

17 Address: Psychology, University of Southampton, Southampton, SO17 1BJ, UK.

9 
Abstract

Only a subset of visual signals give rise to a conscious percept. Threat signals, such as fearful faces, are particularly salient to human vision. Research suggests that fearful faces are evaluated without awareness and preferentially promoted to conscious perception. This agrees with evolutionary theories that posit a dedicated pathway specialised in processing threat-relevant signals. We propose an alternative explanation for this "fear advantage". Using psychophysical data from continuous flash suppression (CFS) and masking experiments, we demonstrate that awareness of facial expressions is predicted by effective contrast: the relationship between their Fourier spectrum and the contrast sensitivity function. Fearful faces have higher effective contrast than neutral expressions and this, not threat content, predicts their enhanced access to awareness. Importantly, our findings do not support the existence of a specialised mechanism that promotes threatening stimuli to awareness. 
An important predictor of survival is the ability to detect threat. However, given the

capacity limits of our sensory systems, not all visual inputs give rise to a conscious percept many stimuli within our field of view go undetected in the competition for neural resources (Dehaene \& Changeux, 2011). How does a limited-capacity system selectively process those inputs of most significance for survival? A widely held view is that humans have a specialised, subcortical visual pathway that expedites the processing of threatening stimuli (Ohman, 2005). An important component of this proposal is that this pathway is thought to operate independently of conscious awareness (Tamietto \& de Gelder, 2010). In the context of survival, it would be advantageous if threats in the environment could influence behaviour before, or without, an observer's awareness of them.

Evidence that threat can be processed preconsciously, or without awareness, comes from paradigms in which visual input is dissociated from awareness (Kim \& Blake, 2005). In backward masking, awareness of a briefly presented image is restricted by the subsequent presentation of a co-located mask. Neuroimaging evidence indicates that masked fearful faces can increase amygdala activity, which is indicative of emotional arousal (Whalen et al., 1998; Whalen et al., 2004). Behaviourally, an observer's response to a peripheral "probe" stimulus is faster when preceded by a masked fearful face than a masked neutral face (Carlson \& Reinke, 2008; Fox, 2002). Continuous flash suppression (CFS) is a technique in which a stable image shown to one eye is suppressed from perception by a dynamic stream of images presented to the other (Tsuchiya \& Koch, 2005). Fearful faces break into awareness from CFS more quickly than neutral faces (Sylvers, Brennan, \& Lilienfeld, 2011; Yang, Zald, $\&$ Blake, 2007). Together, these findings suggest that fear faces are evaluated without awareness and gain prioritised access to conscious vision. Interestingly, our own metaanalyses show that fearful faces are the only threat stimuli to be reliably prioritised over neutral stimuli across the masked visual probe, binocular rivalry and continuous flash 
suppression paradigms (Hedger, Adams, \& Garner, 2014). Thus, subliminally presented fearful expressions do appear to elicit prioritised processing, relative to other threat stimuli, and this effect warrants careful investigation.

Ecological models distinguish between two components of visual signals: content and efficacy (Guilford \& Dawkins, 1991). The former relates to the "message" of the signal, whereas the latter relates to the efficient transmission of the signal in relation to the sensory biases of an observer. It is often assumed that fearful faces are prioritised in the competition for awareness due to their content, since they signal important information about potential threats (Sylvers et al., 2011; Yang et al., 2007). However, fearful faces may also be prioritised due to their efficacy; fast detection could be mediated by purely low-level factors, such as the high contrast physical signal emanating from the eye region - i.e. the increased exposure of the iris and scleral field (Lee, Susskind, \& Anderson, 2013). This latter position is in-line with the 'sensory bias hypothesis' (Horstmann \& Ansorge, 2009; Horstmann \& Bauland, 2006), which states that facial expression signals are salient to sensory biases of human observers. Thus, without characterising the sensory properties of facial signals, we risk attributing prioritised detection to threat-sensitive processes, when it may be better explained by the low-level physical salience of the expression.

Here, we consider this dilemma. There are two possible mechanisms via which fear faces, or other threat-relevant stimuli might gain prioritised access to conscious vision. First, humans might have evolved specialised mechanisms that evaluate the threat content of visual signals prior to their conscious registration. Second, the physical expression of fear might exploit the sensory tuning of early, general-purpose visual processing. In this latter case, the apparent "threat advantage" could be parsimoniously explained by sensory efficacy, without the involvement of pre-conscious mechanisms sensitive to threat. 
Typically, these two accounts are conflated, since the low-level characteristics of facial expressions define the content of the communicated emotion (e.g. wide eyes signal fear). To resolve this issue, therefore, one must experimentally dissociate a stimulus' sensory and affective properties. In the present study, we addressed this issue with a combination of image analyses and behavioural data. First, we use known properties of early visual processes to estimate the efficacy with which emotional expressions are received by human observers. Second, we use stimulus manipulations that modulate the threat content of our images, without affecting sensory efficacy. Third, we present behavioural data from CFS and masking paradigms that quantify the extent to which emotional expressions gain access to awareness. Lastly, we determine whether this is better predicted by (i) the images' low-level, effective contrast (a quantity indifferent to threat), or (ii) their threat-content.

\section{Image Analyses}

Stimulus detection is influenced by low-level properties such as luminance and contrast. Moreover, humans are more sensitive to contrast at certain spatial frequencies, as defined by their contrast sensitivity function (De Valois \& De Valois, 1990). Thus, differences in detection between stimuli (e.g. fearful vs. neutral faces) that differ in these low-level properties cannot be considered a valid measure of threat-related processing. The contrast sensitivity function can be conceptualised as a modulation transfer function for spatial contrast energy at early visual stages. Measuring the extent to which stimuli exploit this sensitivity thus provides an estimate of their sensory efficacy. We asked whether fearful and neutral expressions differ in the extent to which they exploit the contrast sensitivity function (i.e. do they differ in "effective contrast"?).

\section{Effective Contrast}


We analysed the NimStim face set, a collection of face stimuli that is widely used in studies of emotion recognition and is subsequently employed in our behavioural experiments. The set includes 24 male and 19 female models from multiple ethnicities (Tottenham et al., 2009). First, we applied an opaque elliptical mask to eliminate external features before equating mean luminance and root mean squared contrast (RMS) of the images (following standard practice in psychophysical experiments). For our initial analyses, we mirrored the average size (13.5 cm bizygomatic diameter, see Katsikitis, 2003) and a typical distance $(220 \mathrm{~cm})$ of a human face during social interactions.

To calculate effective contrast we followed the procedure of Baker and Graf (2009), implemented in MATLAB (Mathworks). This measure of effective contrast has previously been found to be a good predictor of stimulus salience in binocular rivalry competition (Baker \& Graf, 2009). We obtained the amplitude spectrum (contrast energy as a function of spatial frequency) of each face image (figure 1a, left panel). We then fitted a second order polynomial to the contrast sensitivity data set "ModelFest" (Watson \& Amuhada, 2005) to obtain a continuous contrast sensitivity distribution (figure 1a, middle panel, normalised to the $0-1$ range). By multiplying this distribution by the amplitude spectrum, we obtained effective contrast as a function of spatial frequency, for each stimulus (figure 1a, right panel). Summing this contrast across spatial frequency produces an overall estimate of contrast energy after attenuation by the contrast sensitivity function, i.e. the image's effective contrast. Fear faces had higher effective contrast than neutral faces for 41 of the 43 models, and this difference was large in magnitude $(d=0.76,95 \%$ CI [0.31 1.21], $p<.001)$. To confirm that this finding generalised beyond this particular image set, we extended our analysis to 641 images by including 4 other widely used face sets (frontoparallel faces only): the Karolinska Directed Emotional Faces (KDEF: Lundqvist, Flykt, \& Ohman, 1998), The Radboud Faces Database (RaFD: Langer et al., 2010), The Pictures of 
148 Facial Affect Dataset (Ekman \& Friesen, 1976), The Montreal Set of Facial Displays of

149 Emotion (MSFDE: Beaupre \& Hess, 2005). The sensory advantage of fear was large and

150 significant in all but the Ekman \& Friesen $\operatorname{set}^{1}$ (figure 1b). The pooled effect size across face

151 sets, calculated via a random effects model, was large and significant $(k=6, N=641, d=1.00$,

$15295 \%$ CI $[0.691 .31], p<.001)$. Based on this pooled estimate, the "probability of superiority"

153 (Grissom \& Kim, 2005), i.e. the likelihood that a randomly sampled fearful face will have a sensory advantage over a randomly sampled neutral face, is $84 \%$ (95\% CI [ $75 \% 90 \%])$.

\section{Stability Across Viewing Distances}

The effective contrast differences described above are not scale-invariant; they depend on the particular retinal size of the images. If our physical expression of fear is optimised to be salient in everyday social contexts (Gray, Adams, Hedger, Newton, \& Garner, 2013) then this sensory advantage of fear should be robust over distances at which humans typically socialise and communicate. To test this possibility, we extended our analyses to simulated viewing distances of $50-500 \mathrm{~cm}$. As shown in figure $1 \mathrm{c}$, the sensory benefit of fear is largest within interpersonal proximities that characterise human social interactions (120 to $360 \mathrm{~cm}$, region within dotted lines, see Argyle, 2013). 


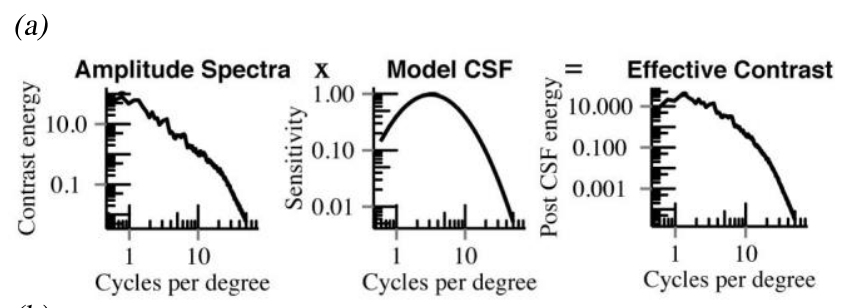

(b)
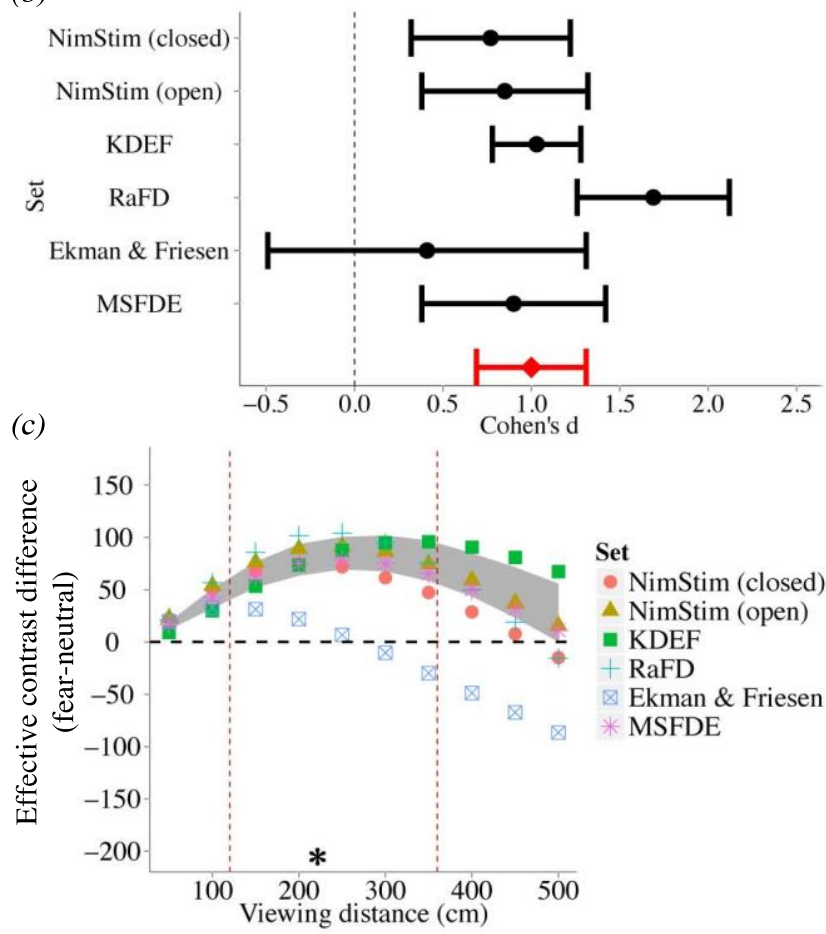

Figure 1. (a) Image analysis for one example fearful face. (CSF = contrast sensitivity

function). (b) Forest plot depicting the effect sizes for effective contrast differences between fearful and neutral faces (open $=$ open mouthed, closed $=$ closed mouthed). Error bars are 95\% confidence intervals. The diamond depicts the pooled effect size. (c) The difference in effective contrast (arbitrary units) between fearful and neutral models as a function of viewing distance. Coloured symbols indicate the mean within each face set, shaded grey region is the bootstrapped $95 \%$ confidence interval. The asterisk indicates the viewing distance used for the initial analyses. Dashed vertical lines span the distances that characterise typical human social interactions.

Importantly, our measure of effective contrast is derived from "classic" contrast sensitivity data (i.e. from challenging threshold conditions). It could be argued, therefore, that 
most normal (non CFS or unmasked) viewing conditions are suprathreshold, to which the shape of this threshold contrast sensitivity function may not apply. Indeed, contrast matching experiments have found that perceived suprathreshold contrast is largely invariant with spatial frequency (Georgeson \& Sullivan, 1975). However, as De Valois and De Valois (1990) note, contrast matching is not a direct sensitivity measurement and as such, one cannot conclude what the suprathreshold contrast response function is for different spatial frequencies. In fact, other measures, such as magnitude estimation, show that the high and low frequency attenuation of the contrast sensitivity function is maintained at suprathreshold levels (Cannon, 1979). It is therefore inappropriate to conceptualise the contrast sensitivity function as an epiphenomenon restricted to threshold conditions. By extension, the detection of stimuli in natural viewing conditions can be understood, at least to a first approximation, in terms of the properties of the contrast sensitivity function.

Our analyses suggest that fearful expressions are optimised to excite the early visual processes of proximal observers: fear faces contain greater contrast energy at the spatial frequencies that humans are sensitive to, relative to neutral faces. This advantage is purely sensory, and generalises across gender and race. This sensory advantage could be either an evolutionary or learned adaptation.

The case for an unconscious processing advantage for threatening stimuli is most often evidenced by the prioritisation of fearful over neutral expressions. However, angry faces, although also signalling threat, have typically yielded smaller effects (Hedger, Adams, \& Garner, 2014) and in some cases a disadvantage has been found for angry faces, relative to neutral faces in CFS paradigms (Gray, et al., 2013). Moreover, happy faces, although not signalling threat, have also been found to be prioritised over neutral stimuli in detection paradigms (Becker, Anderson, Mortensen ,Neufeld \& Neel, 2011) and there is some evidence that they are processed subliminally (Dimberg, Thunberg, \& Elmehed, 2000; Schupp et al., 
2004). Given the inconsistent nature of these findings, it is important to understand whether processing differences between these expressions are better explained by their sensory or affective characteristics. To this end, we used CFS (Experiment 1) and masking paradigms (Experiment 2) to investigate whether effective contrast can predict conscious perception of fearful, angry, happy and neutral faces.

\section{Behavioural Experiment: Access to Awareness from CFS}

Under most viewing conditions, our two eyes receive slightly different views of the world and we perceive a single "fused" percept (Howard \& Rogers, 1995). However, when dissimilar images are presented to our two eyes at corresponding retinal locations, conscious perception alternates between the two images as their neural representations compete for awareness (Blake \& Logothetis, 2002)- a phenomenon termed binocular rivalry. In some respects, this is a controlled phenomenon that can be used to mimic aspects of natural vision, which involves selection amongst multiple sensory inputs, which are assigned to or omitted from conscious perception. In CFS, a dynamic masking pattern is presented to one eye, which can render a stimulus presented to the other eye invisible for seconds before it breaks suppression and enters conscious awareness (Tsuchiya \& Koch, 2005). The length of this initial suppression has been used as an index of the unconscious salience of the supressed image (the bCFS paradigm, Stein \& Sterzer, 2014). Here, we use this bCFS paradigm to measure the extent to which stimuli gain access to conscious perception.

\section{Methods}

We selected 4 NimStim models, on the basis of their high emotional validity (mean expression recognition accuracy was $87 \%$ - see Tottenham et al., 2009), portraying fearful, happy, angry and neutral expressions. Stimuli subtended $6.2 \times 4.1$ degrees of visual angle (DVA) at the viewing distance of $60 \mathrm{~cm}$ on a $1280 \times 1024$ pixel resolution, gamma corrected 
monitor. In order to decouple our images' low-level, effective contrast from their affective properties, we presented the face stimuli in two different conditions (figure 2a). Normal faces were presented upright with veridical contrast polarity. Control faces were rotated 180 degrees with reversed contrast polarity, producing an image similar to a photographic negative. Together, these manipulations severely disrupt the recognition and affective evaluation of facial expressions (Gray et al., 2013). Critically, however, they do not alter effective contrast ${ }^{2}$. Thus, if the threat or valence of face images is the critical factor in driving access to awareness, we would expect any threat advantage to be reduced or eliminated for the control images. Conversely, if effective contrast is the key predictor for a 'threat advantage', then a similar advantage for the fear expression should be observed within normal and control stimuli.

The trial sequence is shown in figure $2 \mathrm{~b}$. A central fixation cross was presented to each eye via a mirror stereoscope for 1 second. Subsequently, observers viewed a CFS display for 800 milliseconds, during which one eye viewed a pair of dynamic masking patterns and the other viewed a face stimulus at 1.4 DVA to the left or right of fixation. The masking patterns were randomly generated ellipses. This ensured that suppression was not biased towards any particular orientation or spatial frequency. Face stimuli were introduced gradually by linearly increasing RMS contrast from $0-100 \%$ over the 800 millisecond period. Each eye's display was framed by a random dot surround (9.5 x 11.4 DVA) to assist binocular alignment. Temporal frequency influences the strength of CFS suppression, with mask frequencies above $10 \mathrm{~Hz}$ exerting weaker suppression than those below (Yang \& Blake, 2012). We therefore used a $20 \mathrm{~Hz}$ mask to ensure that faces broke suppression on a substantial proportion of trials. After the CFS presentation, observers were required to make a forced choice decision as to whether "anything other than the mask" was visible during the trial. 
This unspeeded measure does not measure response times, or recognition of the target stimulus, which are susceptible to criterion effects (Stein \& Sterzer, 2014)

Twenty-two undergraduate students completed 256 experimental trials (4 expressions (anger, happy, fear, neutral) x 2 stimulus manipulations (normal, control) x 32 repetitions), balanced across face location (left or right of fixation). Our sample size provides in excess of $95 \%$ power to detect a large effect size (Cohen's $d=1.15$, the magnitude of difference in detection between fearful vs. neutral faces from a similar CFS paradigm- Yang et al., 2007).

\section{Results}

The percentage of CFS trials in which face stimuli became visible is shown in figure 2c. Visibility was modulated by expression $(F(3,63)=5.33, p=.002)$ with fear faces visible most often, followed by happy, neutral, then angry faces. It is notable that angry faces were detected least often, as this conflicts with the notion that threat is selectively prioritised. Pair-wise comparisons revealed fear and happy faces were both detected more frequently than angry faces $(p s<.05)$. In addition, stimulus manipulation strongly modulated visibility $(F(1,21)=33.31, p<.001, d=1.06,95 \% C I[0.571 .54]):$ normal faces $(M=50.56, S E=$ 5.11) were detected more frequently than control faces $(M=26.85, S E=4.34)$. Critically, expression and manipulation did not interact in their effects on visibility $(F(3,63)=0.18, p=$ $.905)$ : the main effect of expression was similar for both the normal $(F(3,63)=3.14, p=$ $.031)$ and control $(F(3,63)=3.00, p=.028)$ stimuli, with fear detected most often, followed by happy, neutral and anger in both cases. Importantly, this means that the same modulation of visibility by expression and the same "fear advantage" was observed with control stimuli, whose emotional content severely disrupted. Thus, some physical property, that is unaffected by the stimulus manipulations, must be driving the effect of expression. 
Does effective contrast predict visibility? Visibility was closely related to effective contrast (figure 2d) and a linear regression across the 16 facial models revealed that this was significant, $R^{2}=.301, p=.026$.

Importantly, however, the main effect of stimulus manipulation (normal vs. control) cannot be explained by low-level properties, as the two stimulus categories have equivalent effective contrast. The mechanisms that govern visual awareness may therefore discriminate faces from non-faces (Stein, Sterzer, \& Peelen, 2012), but we found no evidence that emotion or threat had an effect on detection beyond that explained by basic low-level variability between expressions.

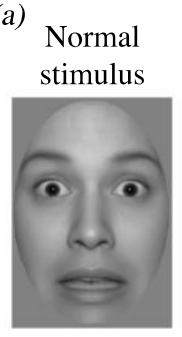

(c) 70

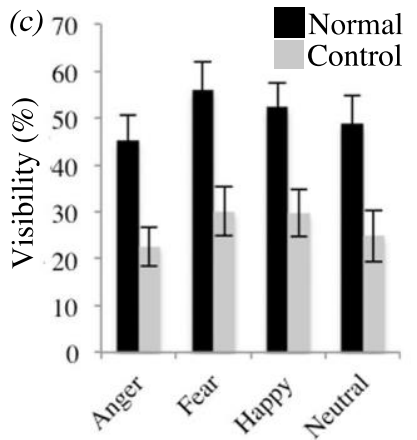

Expression

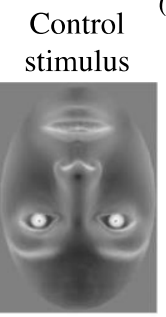

(b) Fixation (1sec)

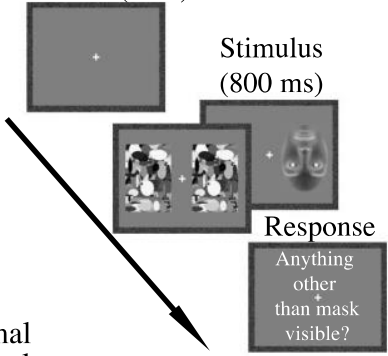

(d)
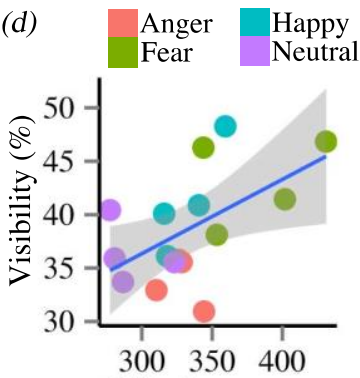

Effective contrast

Figure 2. Experiment 1. (a) Examples of normal and control stimuli. (b) Schematic of CFS trial sequence. (c) Stimulus visibility in the CFS task, as a function of expression and stimulus manipulation. Error bars are \pm 1 SEM. (d) Stimulus visibility as a function of effective contrast, collapsed across manipulation, shaded region is $\pm 1 S E M$. 
The bCFS paradigm has been widely used to investigate the competition for visual awareness. However, we might question whether this represents a naturalistic example of how stimuli compete for awareness; binocular rivalry is infrequently encountered in daily life (Arnold, 2011). In contrast, backward masking involves conditions more typical of everyday vision; observers frequently encounter brief glimpses of stimuli when sampling dynamic scenes via saccades and fixations. In our second experiment, therefore, we investigated whether effective contrast can predict the detection of briefly presented, masked facial expressions. In addition, we asked observers to provide affective ratings of the face stimuli, allowing us to assess the contributions of (i) low-level contrast and (ii) affective factors in stimulus detection.

\section{Method}

Figure 3 shows the masking paradigm. At the beginning of each trial, observers viewed the fixation cross for one second. Next, two masks were presented either side of fixation for $200 \mathrm{~ms}$, followed by a target (intact) and non-target (block-scrambled) face for a variable duration $(13.3,26.6,40,53.3,66.7,80,93.3,106.7$ or $120 \mathrm{~ms})$. Subsequently, two new mask stimuli were presented for $200 \mathrm{~ms}$, immediately following the face presentation. Participants were asked to indicate, as accurately as possible, whether the intact face had appeared to the left or right of fixation.

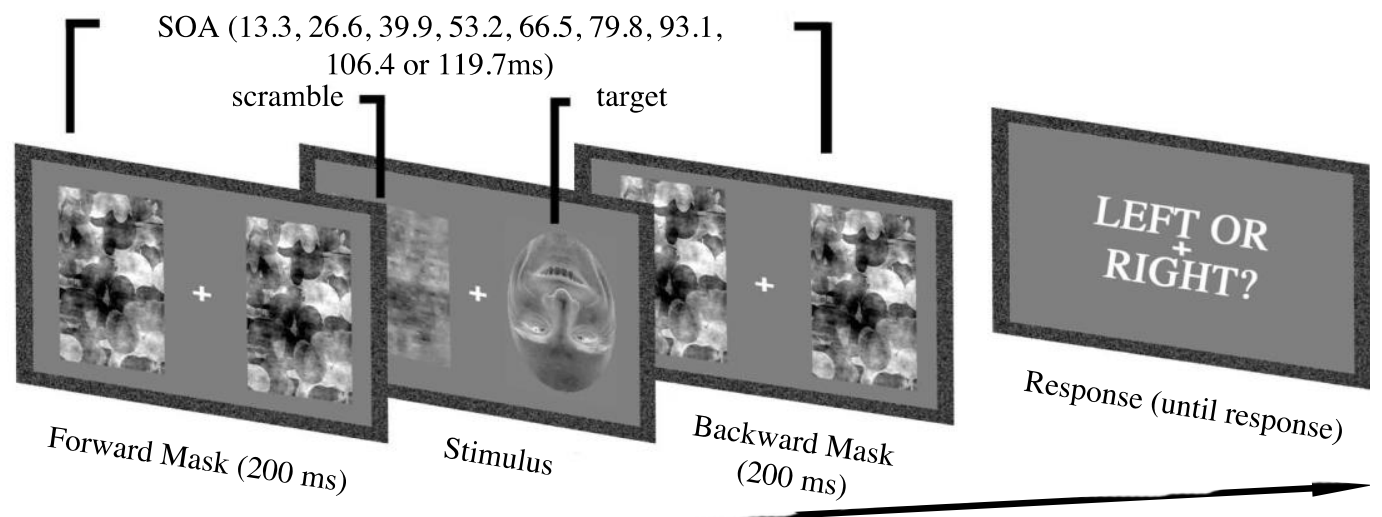


Figure 3. Schematic of trial sequence in the masking task.

All stimulus dimensions matched those in Experiment 1. The scrambled face matched the amplitude spectrum averaged across all target face stimuli, ensuring the target could not be localised via non-specific differences in luminance or contrast between the two sides of the display. Mask stimuli also matched the averaged spectral slope of all face stimuli. This prevented interactions between the spatial frequency profile of the target and mask from influencing detection.

An independent sample of 11 participants completed 1152 randomly ordered trials (4 expressions (anger, fear, happy, neutral) x 2 stimulus manipulations (normal, control) x 9 stimulus onset asynchronies (SOAs: 13.3, 26.6, 40, 53.3, 66.7, 80, 93.3, 106.7 or $120 \mathrm{~ms}$ ) x 16 repetitions), balanced across the location of the face stimulus (left, right). Our sample size provided in excess of $95 \%$ power to detect the same target effect size as defined for experiment 1 .

Observers also completed a Self-Assessment Manikin (SAM) to evaluate face stimuli on the three dimensions of emotional assessment: valence, arousal and dominance (see Bradley \& Lang, 1994). On each trial, observers initiated face presentation, which was displayed (unmasked) for 120ms (maximum SOA in the masking task). Valence, arousal and dominance ratings (1-9 scale) were made in separate blocks, consisting of 32 randomly ordered trials.

\section{Results}

Following standard practice, valence and arousal ratings are summarised in "affective space" (figure 4a). For normal faces, the distribution of stimuli follows the expected “boomerang” shape (Bradley, Codispoti, Cuthbert, \& Lang, 2001) with higher arousal levels reported for stimuli with large positive or negative valence. However, no such pattern is 
visible for the control stimuli. A cluster analyses confirms this - the distribution of normal stimuli is optimally explained (as determined by Bayesian Information Criterion) by a 3 cluster model that clearly differentiates between the positive (happy) negative (fear, anger) and neutral (neutral) expressions. In contrast, the distribution of control stimuli is optimally explained by a one-cluster model; expressions are not differentiated in affective space. This, consistent with previous work (Gray et al., 2013) confirms that our stimulus manipulations of spatial and contrast inversion severely alter the emotional evaluation of facial expressions. It is possible that increased statistical power may have detected residual discrimination (Reingold \& Merikle, 1988). Nonetheless, it is clear from figure 4a that control stimuli elicit a qualitatively different pattern of affective evaluations. Thus, if affective dimensions are important in prioritising emotional stimuli, this difference should be reflected in a different pattern of detection across expression for control stimuli vs. normal stimuli.

Figure $4 \mathrm{~b}$ displays the $2 \mathrm{AFC}$ performance accuracy from the masking task. Data were fitted with cumulative normal distributions free to vary in position and slope. Detection thresholds were estimated from these fits for $75 \%$ correct performance (upper binomial limit). Thresholds were significantly and substantially modulated by stimulus manipulation, with observers requiring longer SOAs to detect control faces than normal faces (normal: $M=$ $60.6 \mathrm{~ms}$, control: $M=84.6 \mathrm{~ms}, t(10)=10.7, p<.001, d=2.54,95 \%$ CI [1.38 3.69]). Notably, these detection thresholds correspond to much briefer stimulus exposures ( $72 \mathrm{~ms}$ on average) than those at which observers made affective judgements in the SAM task (120 ms). Thus, we expect that discrimination of expressions would have been even worse under conditions that are sufficient for any fear advantage. Similarly to Experiment 1 there was a main effect of expression on detection in both the normal $(F(3,30)=9.95, p<.001)$ and control conditions $(F(3,30)=9.22, p<.001)$. This effect was again similar in the two conditions, with no interaction between expression and stimulus manipulation $(F(3,30)=1.15, p=$ 
.345). In other words, although spatial and contrast inversion inhibited emotional recognition of the control stimuli, this did not affect the 'fear advantage' for detection. Normal and control fearful faces were detected at shorter SOAs than both neutral and angry faces $(p s<$. 05, pairwise comparisons). Figure $4 \mathrm{c}$ illustrates the relationship between effective contrast and detection threshold, for all stimuli. Effective contrast was a similarly good predictor of detection thresholds in both the normal $\left(R^{2}=0.36, p=.014\right)$ and control $\left(R^{2}=0.41, p=.004\right)$ configurations.

(a)

Normal Anger Fear

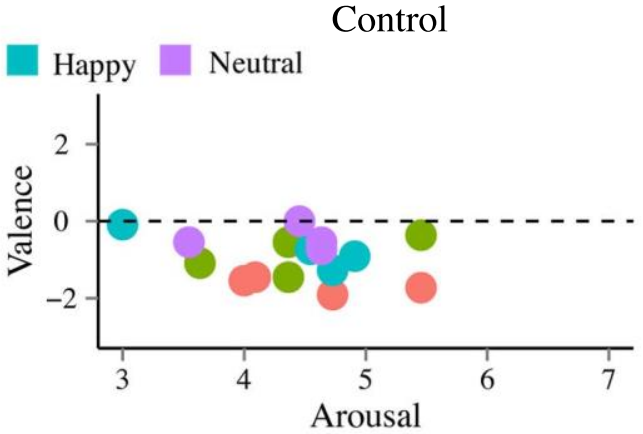

(b)

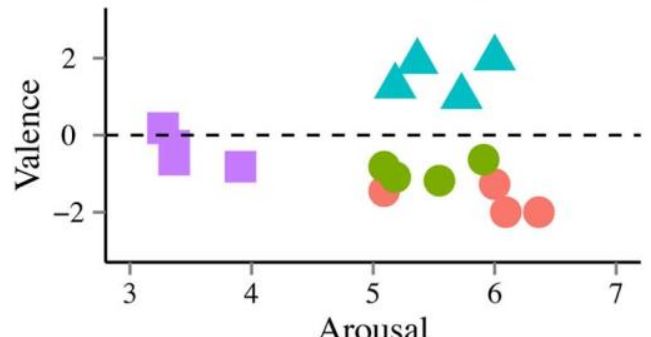

Arousal
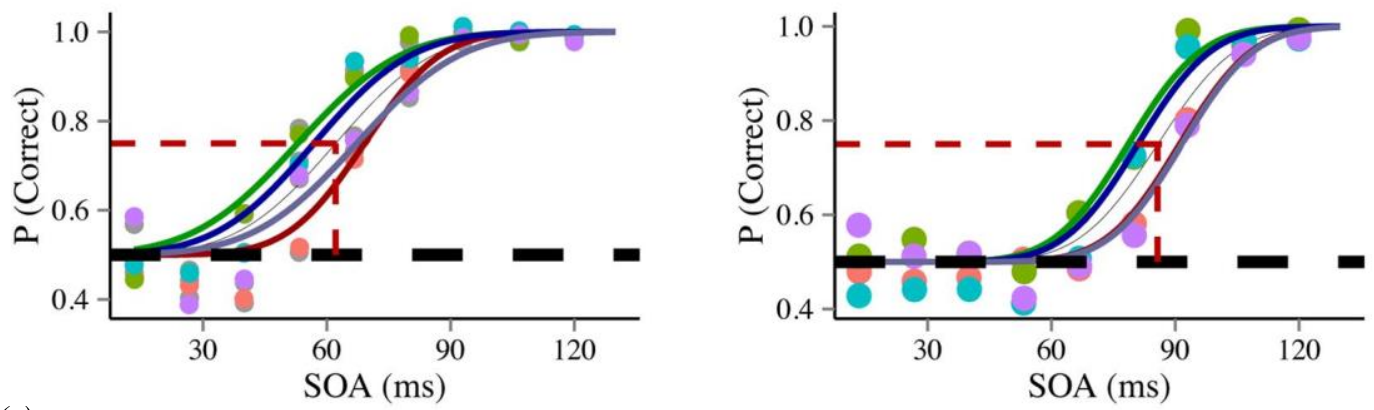

(c)
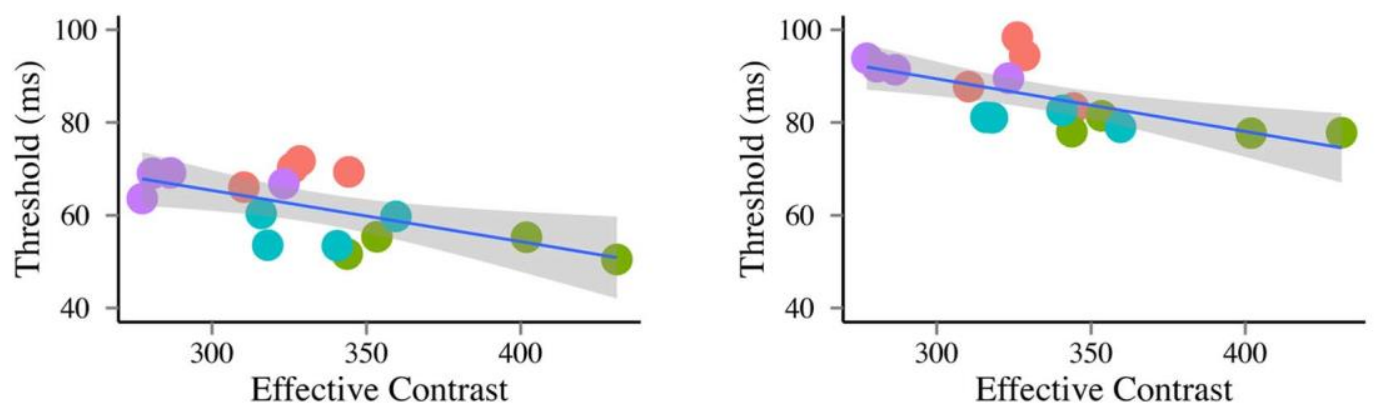

Figure 4. (a) Distribution of stimuli in affective space, according to valence and arousal ratings. Valence is normalised to a -4 to +4 range such that 0 indicates neutral. Symbol colour 
396

represents facial expression, symbol shape represents clusters obtained via Bayes criteria (i.e. normal $=3$ clusters, control $=1$ cluster). $($ b) The proportion of correct face localisation responses is plotted as a function of SOA and expression, with cumulative normal fits. The dashed red lines indicate the mean thresholds for normal and control stimuli (c) Detection threshold as a function of effective contrast. Shaded region indicates $\pm 1 S E M$.

Interestingly, adding expression as a predictor significantly increased the variance explained by effective contrast alone (normal: $F(3,11)=15.96, p<.001$, control: $F(3,11)=$ $5.60, p=.014)$. However, as we have discussed, it is important not to conflate 'expression' (the physical signal) with 'emotion' (i.e. a semantic or affective evaluation of the signal). Thus, the fact that expression improves the model fit simply means that effective contrast cannot entirely account for the effect of expression. Other, unspecified stimulus attributes may also play a role; it does not, in itself, imply an influence of emotion sensitive processes on detection. To strengthen this conviction, we performed regression analyses with valence, arousal and dominance as predictors of thresholds. For both the normal and control configurations, none of these variables significantly predicted detection thresholds (all ps $>.05)$. Notably, the same was true when these affective ratings were used as predictors of the bCFS visibility data from Experiment 1 . Moreover, tests for zero partial association revealed that the relationship between effective contrast and detection thresholds remained significant after controlling for the influence of all these variables (normal: $t(11)=-2.57, p=.026$, control: $t(11)=-3.47, p=.005)$. In summary, we found that low-level effective contrast predicts stimulus visibility, but found no effect of emotion sensitive processes on detection. 
Our data establish that global differences in effective contrast can predict the prioritisation of faces in the competition for awareness. However, we can refine our analyses further to ask whether this is driven by particular image regions. These regions were defined by symmetric Gaussian windows whose size and standard deviation (2 DVA, 0.5 DVA respectively) matched the stimuli used to derive the Modelfest data (Watson \& Amuhada, 2005). These windows were applied to 130 uniformly distributed, overlapping locations within each image and effective contrast was calculated for each region. The relative (z scored) effective contrast, averaged across the models used in our experiments is shown in figure 5. Consistent with previous suggestions (Gray et al., 2013; Lee et al., 2013) it is clear that the eye region is highly salient within all expressions, but particularly so for fear faces. This can be attributed to the increased exposure of the white scleral field and dark iris. These features are unique amongst primates and probably co-evolved with human social communication to enhance detectability of gaze (Kobayashi \& Kohshima, 1997). Expressing fear amplifies this sensory benefit by increasing the vertical dimension of the scleral field (see Hedger, 2014 for a demonstration).

Figure 5. Local variations in effective contrast. Image colour/luminance represents the Zscored effective contrast.

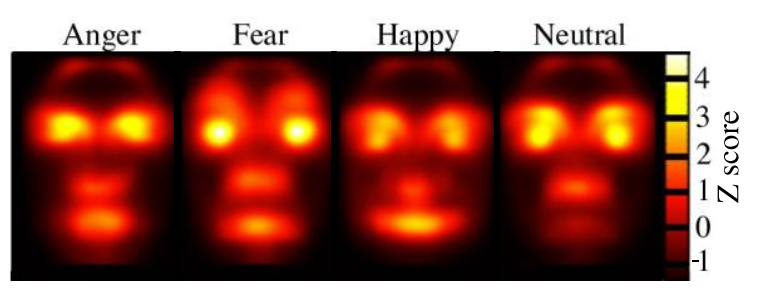

\section{Discussion}

Shaped by sociobiological pressures, human signals are designed to reliably convey information to observers. In the context of threat, a sender may express fear to warn others of 
danger, or to signal appeasement. However, before a signal can be acted upon, it must be detected. Theories of enhanced signal function by design (Dukas, 1998) thus predict that the facial expression of fear would converge on a form that exploits the sensory processes of a proximal observer. By analysing the Fourier content of faces in the context of human interactions, we found that facial expressions differ in the extent to which they stimulate lowlevel visual processes. This mechanism, effective contrast, provides a parsimonious explanation for the prioritisation of fearful faces in the competition for awareness, across rather different paradigms: CFS and masking. Critically, this 'threat advantage' was poorly explained by perceived emotion; it was unchanged for stimuli with the same effective contrast, but altered emotional content. Moreover, face detection was not predicted by observers' affective ratings. Our data are inconsistent with the notion that the threat value of fear faces is evaluated outside of awareness and determines access to conscious vision. Instead, our data suggest that access is best explained by the tuning of very early visual processes, i.e. the contrast sensitivity function.

Previous work has speculated that the prioritised detection of threat relevant stimuli (including fearful faces) may be linked to simple, low-level stimulus properties (Bar, \& Neta, 2006; Coelho, Cloete, \& Wallis, 2010; Gray et al., 2013; Stein \& Sterzer, 2012; Lee et al., 2013; Yang et al., 2007). However, these studies have not quantified these properties with respect to underlying human sensory processes. Moreover, to our knowledge, our study is the first to explicitly quantify both sensory (effective contrast) and affective properties (SAM) of facial expressions as predictors in a detection paradigm. We found that low-level sensory properties were by far the best predictor of stimulus detection and found no evidence that detection was modulated by threat-sensitive processes. The data thus suggest that the fear advantage is most parsimoniously explained by low-level properties of the fear expression, negating the need to invoke the role of threat, or emotion sensitive processes. 
Detectability is unlikely to be the only factor that has shaped the fear expression.

Expressing fear enhances the expresser's field of view, eye movement velocity and nasal

airflow- linking it to functional advantages in the context of threat (Susskind et al., 2008).

Fear may also enhance the expresser's stimulus detection by shaping how light is cast onto their retina (Lee, Mirza, Flanagan, \& Anderson, 2014). In other words, the expression of fear reception of visual information. appears to be adaptive for both senders and receivers, in terms of efficient transmission and

We observed a robust "face advantage" in both experiments. Normal faces were better detected than control faces, despite being equivalent in effective contrast. This suggests that the visual system is sensitive to stimuli that are specifically face-like, and this sensitivity is not yoked to awareness of the stimuli. It has been found in bCFS studies that stimulus inversion has a detrimental effect on the detection of human faces but no effect on detection of chimpanzee faces (Stein, et al., 2012). This suggests that pre-conscious visual processes selectively promote stimuli that resemble conspecific faces to conscious perception, presumably because of their social relevance. However, our data suggest that this sensitivity does not extend to the evaluation of facial emotion; emotional evaluations had no effect on stimulus detection beyond that explained by low-level image properties.

How can we reconcile a robust face advantage with the absence of emotion-sensitive processes? Determining whether a stimulus is a face represents a coarser-level judgement than identifying its emotional expression. Visual masking studies have shown that identifying a specific object requires substantially more processing time than identifying its general category, whilst determining an object's category co-occurs with its detection (Grill-Spector \& Kanwisher, 2005). The present data are thus consistent with a framework in which the detection and categorisation of faces (i.e. face vs. non face), but not the evaluation of facial expression, is performed at an early processing stage by the same perceptual mechanisms. In 
contrast, the data are poorly explained by models suggesting that emotional evaluation precedes and drives face detection (Palermo \& Rhodes, 2007).

It is important to consider well-documented phenomena that appear to conflict with our "low-level" account of the fear advantage. One relevant example is that anxious populations exhibit enhanced processing of fear faces, which is commonly attributed to dysfunction in threat-sensitive mechanisms that operate without awareness (Bar Haim, Lamy, Pergamin, Bakermans-Kranenberg, \& van IJzendoorn, 2007). However, differences in perceptual selection observed in anxious populations are not limited to threat relevant contexts. For instance, anxiety is associated with enhanced attentional capture by perceptually salient neutral stimuli (Moser, Becker, \& Moran, 2012, Moran \& Moser, 2014). Correspondingly, enhanced biases for fear faces in anxious populations could be a function of either the perceptual or emotional properties of the stimuli. Thus, processing differences displayed by anxious populations may not be inconsistent with our account.

Another interesting phenomenon is that eye gaze direction can modulate detection of fearful faces, such that averted fearful gazes are prioritised over directed fearful gazes (Milders, Hietan, Leppanen, \& Braun, 2011). This makes good ecological sense in terms of perceived threat, since the presence of an unknown threat in the environment (averted gaze) may be more dangerous than a threat directly from the target (directed gaze). Importantly, however, Chen and Yeh (2012) found directly contradictory results using schematic faces, in which low-level variability is reduced. In a bCFS paradigm, Chen and Yeh found that schematic fearful faces with directed gaze were detected faster than those with averted gaze. Notably, the removal of the salient eye white in schematic stimuli also resulted in a lack of an overall "fear advantage" for detection. These opposing findings, likely due to simple physical variations between the particular stimulus sets employed, pose problems for accounts that 
posit specialised threat detection mechanisms as the cause of processing biases (see Becker, et al., 2011 for a related discussion).

Several studies have observed differential amygdala responses to fearful and neutral faces rendered invisible by masking and CFS (Jiang \& He, 2006; Whalen et al., 2004), which has been interpreted as evidence that fearful faces are evaluated without awareness via a pathway involving the amygdala. However, whether this neural activity is linked to adaptive changes in perception is hard to determine without convergent behavioural measures.

Importantly, patients with amygdala lesions nonetheless show prioritised detection of fear in bCFS and visual search tasks (Piech et al., 2010; Tsuchiya, Moradi, Felsen, Yamakazi, \& Adolphs, 2009). Moreover, recent work using a CFS paradigm suggests that attentional orienting to threat stimuli may be dependent on their conscious detection (Hedger, Adams, \& Garner, in press). Whether amygdala activity to unconsciously presented threat stimuli (in response to either low-level or affective properties) has a functional role in promoting their detection, therefore, remains an interesting question.

How do our data fit with suggestions that processing of threatening stimuli is driven by evaluation of content in the low spatial frequencies? (Mermillod, Droit-Volet, Devaux, Schaefer, \& Vermeulen, 2010; Willenbockel, Leopre, Nguyen, Bouthillier, \& Gosselin, 2012). Such observations are thought to support the notion that coarse, rapid, magnocellular input to the amygdala is sufficient for the evaluation and subsequent detection of threat stimuli (Tamietto \& de Gelder, 2010; Vuileumier, Armony, Driver, \& Dolan, 2003). Importantly, human contrast sensitivity is greater for low spatial frequencies, i.e. they have more influence on our measure of effective contrast. Thus, our data also suggest that low spatial frequencies are important, but that this relates to the distribution of contrast at these spatial scales, not the semantic content that is available, or evaluated at these scales. 
In summary, our data suggest that, through evolutionary or learned adaptations, fearful faces are optimised to stimulate human sensory biases. This mechanism may provide a parsimonious explanation of the "fear advantage" in the competition for awareness that negates the need to invoke preconscious processes sensitive to threat.

3

4

5

.

7

8

9

0

Argyle, M. (2013). Bodily Communication. London: Routledge.

Arnold, D. H. (2011). Why is binocular rivalry uncommon? Discrepant monocular images in the real world. Frontiers in Human Neuroscience, 5. doi:10.3389/fnhum.2011.00116

Baker, D. H., \& Graf, E. W. (2009). Natural images dominate in binocular rivalry. Proceedings of the National Academy of Sciences, 106, 5436-5441. doi:10.1073/pnas.0812860106

Bar-Haim, Y., Lamy, D., Pergamin, L., Bakermans-Kranenburg, M. J., \& van IJzendoorn, M. H. (2007). Threat-related attentional bias in anxious and nonanxious individuals: A meta-analytic study. Psychological Bulletin, 133, 1-24. doi:10.1037/00332909.133.1.1

Bar, M., \& Neta, M. (2006). Humans prefer curved visual objects. Psychological Science, 17, 645-648. doi:10.1111/j.1467-9280.2006.01759.x 
Beaupré, M. G., \& Hess, U. (2005). Cross-cultural emotion recognition among Canadian ethnic groups. Journal of Cross-Cultural Psychology, 36, 355-370. doi:10.1177/0022022104273656

Becker, D. V., Anderson, U. S., Mortensen, C. R., Neufeld, S. L., \& Neel, R. (2011). The face in the crowd effect unconfounded: Happy faces, not angry faces, are more efficiently detected in single- and multiple-target visual search tasks. Journal of Experimental Psychology: General, 140, 637-659. doi:10.1037/a0024060

Blake, R., \& Logothetis, N. K. (2002). Visual competition. Nature Reviews. Neuroscience, 3, 13-21. doi:10.1038/nrn701

Bradley, M. M., Codispoti, M., Cuthbert, B. N., \& Lang, P. J. (2001). Emotion and motivation I: Defensive and appetitive reactions in picture processing. Emotion, 1, 276-298. doi:10.1037/1528-3542.1.3.276

Bradley, M. M., \& Lang, P. J. (1994). Measuring emotion: the Self-Assessment Manikin and the Semantic Differential. Journal of Behavior Therapy and Experimental Psychiatry, $25,49-59$.

Cannon, M.W. (1979). Contrast sensation: A linear function of stimulus contrast. Vision Research, 19, 1045-1052

Carlson, J. M., \& Reinke, K. S. (2008). Masked fearful faces modulate the orienting of covert spatial attention. Emotion, 8, 522-529.

Chen, Y.C., \& Yeh, S.-L. (2012). Look into my eyes and I will see you: unconscious processing of human gaze. Consciousness and Cognition, 21, 1703-1710. doi:10.1016/j.concog.2012.10.001

Coelho, C. M., Cloete, S., \& Wallis, G. (2010). The face-in-the-crowd effect: when angry faces are just cross(es). Journal of Vision, 10, 1-14. doi:10.1167/10.1.7 
Dehaene, S., \& Changeux, J.-P. (2011). Experimental and theoretical approaches to conscious processing. Neuron, 70, 200-227. doi:10.1016/j.neuron.2011.03.018

De Valois, K.K. (1977). Spatial frequency adaptation can enhance contrast sensitivity. Vision Research, 17, 1057-1065.

De Valois, R.L., \& De Valois, K.K. (1990). Spatial vision. New York: Oxford University Press.

Dimberg, U., Thunberg, M., \& Elmehed, K. (2000). Unconscious facial reactions to emotional facial expressions. Psychological science, 11, 86-89.

Dukas, R. (1998). Cognitive Ecology: The Evolutionary Ecology of Information Processing and Decision Making. Chicago: University of Chicago.

Ekman, P., \& Friesen, W. V. (1976). Pictures of facial affect. Palo Alto, CA; Consulting Psychologists.

Fox, E. (2002). Processing emotional facial expressions: The role of anxiety and awareness. Cognitive, Affective \& Behavioral Neuroscience, 2, 52-63.

Georgeson, M.A., \& Sullivan, G.D. (1975). Contrast constancy: Deblurring in human vision by spatial frequency channels. Journal of Physiology, 252, 627-656.

Gray, K. L. H., Adams, W. J., Hedger, N., Newton, K. E., \& Garner, M. (2013). Faces and awareness: low-level, not emotional factors determine perceptual dominance. Emotion, 13, 537-544. doi:10.1037/a0031403

Grill-Spector, K., \& Kanwisher, N. (2005). Visual recognition: As soon as you know it is there, you know what it is. Psychological Science, 16, 152-160. doi:10.1111/j.09567976.2005.00796.x

Grissom., \& Kim. (2005). Effect sizes for research: A broad practical approach. New York, NY: Holt, Reinhart, \& Winston. 
Guilford, T., \& Dawkins, M. S. (1991). Receiver psychology and the evolution of animal signals. Animal Behaviour, 42, 1-14. doi:10.1016/S0003-3472(05)80600-1

Haun, A. M., \& Peli, E. (2013). Perceived contrast in complex images. Journal of Vision, 13. doi:10.1167/13.13.3

Hedger, N. Fearfulness and effective contrast. figshare. http://dx.doi.org/10.6084/m9.figshare.1104425

Hedger, N., Adams, W. J., \& Garner, M. (2014). Fearful facial expressions are salient to early visual processes: evidence from effective contrast analyses and continuous flash suppression. Journal of Vision, 14, 1387-1387. http://doi.org/10.1167/14.10.1387

Hedger, N., Adams, W. J., \& Garner, M. (in press). Autonomic arousal and attentional orienting to visual threat are predicted by awareness. Journal of Experimental Psychology: Human Perception and Performance.

Horstmann, G., \& Ansorge, U. (2009). Visual search for facial expressions of emotions: a comparison of dynamic and static faces. Emotion, 9, 29-38

Horstmann, G., \& Bauland, A. (2006). Search asymmetries with real faces: testing the angersuperiority effect. Emotion, 6, 193-207.

Howard, I. P., \& Rogers, B. J. (1996). Binocular Vision and Stereopsis. New York: Oxford University Press.

Jiang, Y., \& He, S. (2006). Cortical responses to invisible faces: dissociating subsystems for facial-information processing. Current Biology, 16, 2023-2029. doi:10.1016/j.cub.2006.08.084

Katsikitis, M. (2003). The Human Face: Measurement and Meaning. Dordecht: Kluwer.

Kim, C.Y., \& Blake, R. (2005). Psychophysical magic: rendering the visible "invisible." Trends in Cognitive Sciences, 9, 381-388. doi:10.1016/j.tics.2005.06.012 
Kobayashi, H., \& Kohshima, S. (1997). Unique morphology of the human eye. Nature, 387, 767-768. doi:10.1038/42842

Langner, O., Dotsch, R., Bijlstra, G., Wigboldus, D. H. J., Hawk, S.T., \& van Knippenberg, A. (2010). Presentation and validation of the Radboud Faces Database. Cognition \& Emotion, 24, 1377-1388. doi: 10.1080/02699930903485076

Lee, D. H., Mirza, R., Flanagan, J. G., \& Anderson, A. K. (2014). Optical origins of opposing facial expression actions. Psychological Science, 0956797613514451. doi:10.1177/0956797613514451

Lee, D. H., Susskind, J. M., \& Anderson, A. K. (2013). Social transmission of the sensory benefits of eye widening in fear expressions. Psychological Science, 24, 957-965.

Lundqvist, D., Flykt, A., \& Öhman, A. (1998). The Karolinska Directed Emotional Faces (KDEF). Stockholm: Karolinska Institute

Mermillod, M., Droit-Volet, S., Devaux, D., Schaefer, A., \& Vermeulen, N. (2010). Are coarse scales sufficient for fast detection of visual threat? Psychological science, 10, 14291437.

Milders, M., Hietanen, J. K., Leppänen, J. M., \& Braun, M. (2011). Detection of emotional faces is modulated by the direction of eye gaze. Emotion, 11, 1456-1461. doi:10.1037/a0022901

Moran, T. P., \& Moser, J. S. (2015). The color of anxiety: Neurobehavioral evidence for distraction by perceptually salient stimuli in anxiety. Cognitive, Affective \& Behavioral Neuroscience, 15, 169-179. http://doi.org/10.3758/s13415-014-0314-7

Moser, J. S., Becker, M. W., \& Moran, T. P. (2012). Enhanced attentional capture in trait anxiety. Emotion 12, 213-216. doi:10.1037/a0026156

Öhman, A. (2005). The role of the amygdala in human fear: Automatic detection of threat. Psychoneuroendocrinology, 30, 953-958. doi:10.1016/j.psyneuen.2005.03.019 
Palermo, R., \& Rhodes, G. (2007). Are you always on my mind? A review of how face perception and attention interact. Neuropsychologia, 45, 75-92. doi:10.1016/j.neuropsychologia.2006.04.025

Piech, R. M., McHugo, M., Smith, S. D., Dukic, M. S., Meer, J. V. D., Abou-Khalil, B., \& Zald, D. H. (2010). Fear-enhanced visual search persists after amygdala lesions. Neuropsychologia, 48, 3430-3435. doi:10.1016/j.neuropsychologia.2010.07.009

Reingold, E. M., \& Merikle, P. M. (1988). Using direct and indirect measures to study perception without awareness. Perception \& Psychophysics, 44, 563-575.

Schupp, H. T., Öhman, A., Junghöfer, M., Weike, A. I., Stockburger, J., \& Hamm, A. O. (2004). The facilitated processing of threatening faces: an ERP analysis. Emotion, 4, 189-200.

Stein, T., \& Sterzer, P. (2012). Not just another face in the crowd: Detecting emotional schematic faces during continuous flash suppression. Emotion, 12, 988-996. doi: $10.1037 / \mathrm{a} 0026944$

Stein, T., \& Sterzer, P. (2014). Unconscious processing under interocular suppression: getting the right measure. Frontiers in Psychology, 5, 387. doi:10.3389/fpsyg.2014.00387

Stein, T., Sterzer, P., \& Peelen, M. V. (2012). Privileged detection of conspecifics: evidence from inversion effects during continuous flash suppression. Cognition, 125, 64-79. doi:10.1016/j.cognition.2012.06.005

Susskind, J. M., Lee, D. H., Cusi, A., Feiman, R., Grabski, W., \& Anderson, A. K. (2008). Expressing fear enhances sensory acquisition. Nature Neuroscience, 11, 843-850. doi:10.1038/nn.2138

Sylvers, P. D., Brennan, P. A., \& Lilienfeld, S. O. (2011). Psychopathic traits and preattentive threat processing in children: A novel test of the fearlessness hypothesis. Psychological Science, 22, 1280-1287. doi:10.1177/0956797611420730 
Tamietto, M., \& de Gelder, B. (2010). Neural bases of the non-conscious perception of emotional signals. Nature Reviews Neuroscience, 11, 697-709. doi:10.1038/nrn2889

Tottenham, N., Tanaka, J. W., Leon, A. C., McCarry, T., Nurse, M., Hare, T. A., ... Nelson, C. (2009). The NimStim set of facial expressions: Judgments from untrained research participants. Psychiatry Research, 168, 242-249. doi:10.1016/j.psychres.2008.05.006

Tsuchiya, N., \& Koch, C. (2005). Continuous flash suppression reduces negative afterimages. Nature Neuroscience, 8, 1096-1101. doi:10.1038/nn1500

Tsuchiya, N., Moradi, F., Felsen, C., Yamazaki, M., \& Adolphs, R. (2009). Intact rapid detection of fearful faces in the absence of the amygdala. Nature Neuroscience, 12, 1224-1225. doi:10.1038/nn.2380

Vuilleumier, P., Armony, J. L., Driver, J., \& Dolan, R. J. (2003). Distinct spatial frequency sensitivities for processing faces and emotional expressions. Nature Neuroscience, 6 , $624-631$.

Watson, A. B., \& Ahumada, A. J. (2005). A standard model for foveal detection of spatial contrast. Journal of Vision, 5, 6. doi:10.1167/5.9.6

Whalen, P. J., Kagan, J., Cook, R. G., Davis, F. C., Kim, H., Polis, S., .. Johnstone, T. (2004). Human amygdala responsivity to masked fearful eye whites. Science, 306, 20612061. doi:10.1126/science. 1103617

Whalen, P. J., Rauch, S. L., Etcoff, N. L., McInerney, S. C., Lee, M. B., \& Jenike, M. A. (1998). Masked presentations of emotional facial expressions modulate amygdala activity without explicit knowledge. The Journal of Neuroscience, 18, 411-418.

Willenbockel, V., Lepore, F., Nguyen, D. K., Bouthillier, A., \& Gosselin, F. (2012). Spatial frequency tuning during the conscious and non-conscious perception of emotional facial expressions-an intracranial ERP study. Frontiers in psychology, 3, 237. 
690 Yang, E., Zald, D. H., \& Blake, R. (2007). Fearful expressions gain preferential access to

691

692

693

694

695

696

697

698

699

700

701

702

703

704

705

706

707

708

709

710

711

712

713

714

09

awareness during continuous flash suppression. Emotion, 7, 882-886. doi:10.1037/1528-3542.7.4.882

Yang, E., \& Blake, R. (2012). Deconstructing continuous flash suppression. Journal of Vision, 12, 8. doi:10.1167/12.3.8

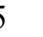

6

697

698

699

00

01

03

04

05

06

707

\section{8}

10 11

12 

relationships between effective contrast and detection remained significant after controlling 739 for skew. 
(\$iguremal stimulus

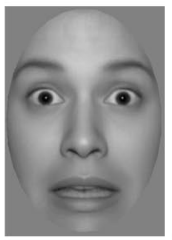

(c) 70
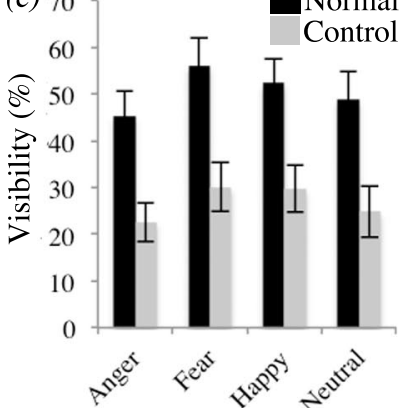

Control stimulus

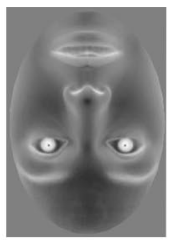

Normal Control

(b) Fixation (1sec)

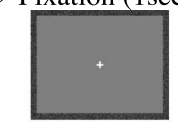

Stimulus

(800 ms)

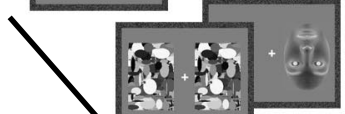

Response

Anything

other

than mask

visible?
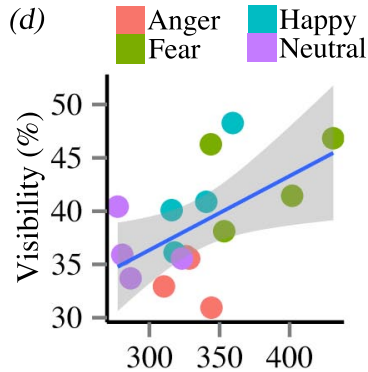

Effective contrast 

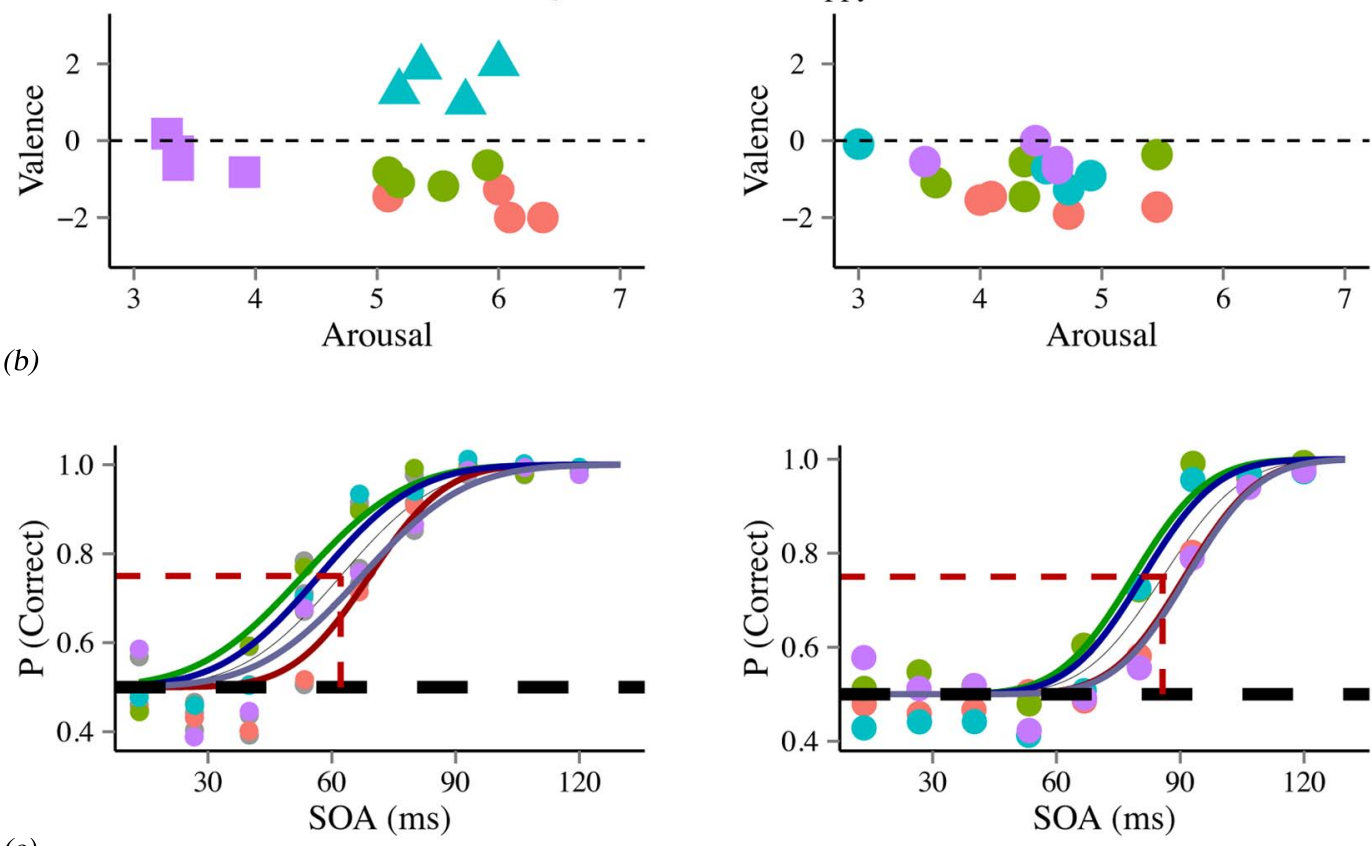

(c)
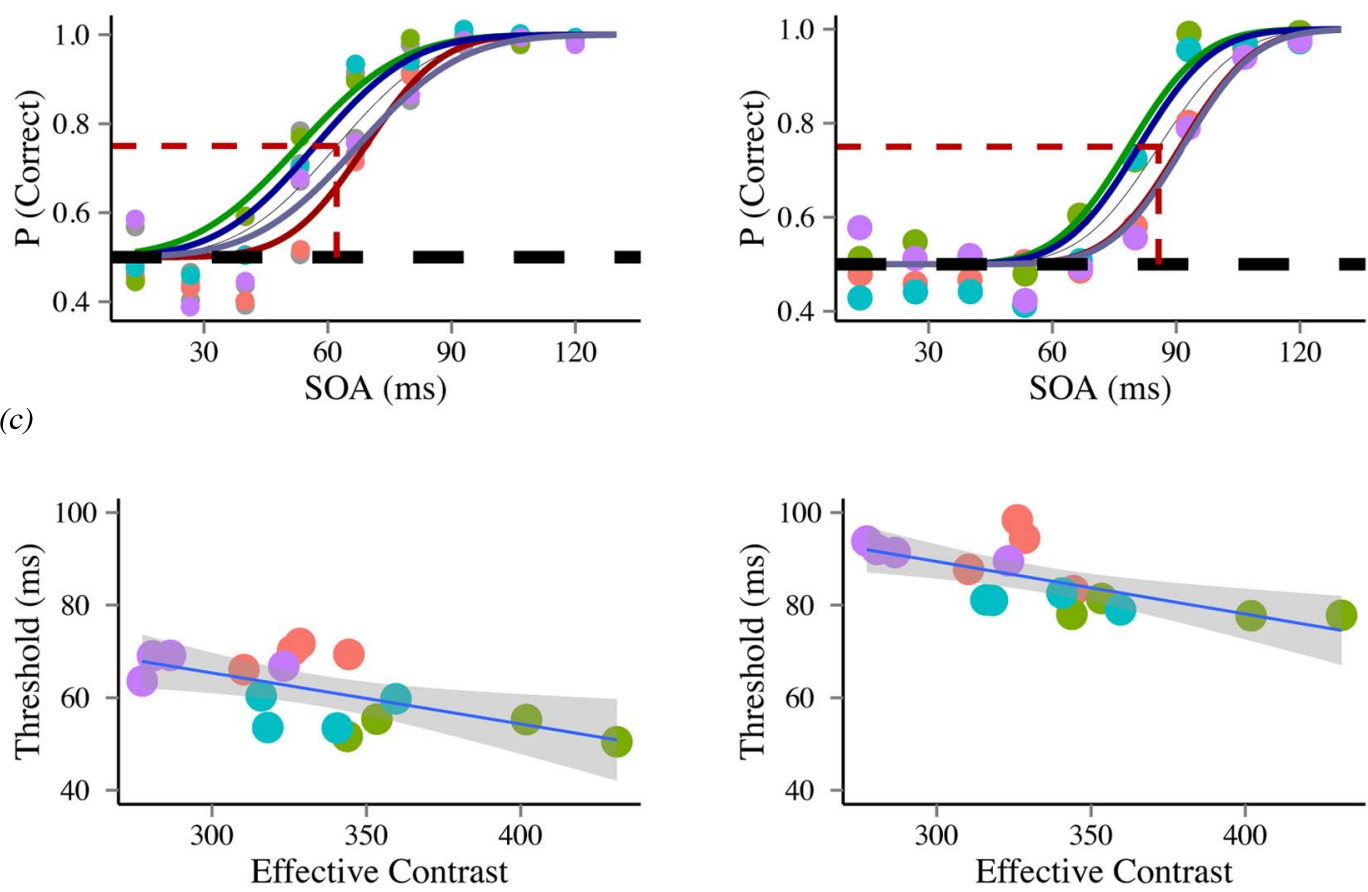
